Meta

Journal des traducteurs

Translators' Journal

\title{
Traitement d'une paire terminologique : Ground Range Image et Slant Range Image
}

\section{Loïc Depecker}

Volume 36, numéro 1, mars 1991

La terminologie dans le monde : orientations et recherches

URI : https://id.erudit.org/iderudit/002538ar

Aller au sommaire du numéro

Éditeur(s)

Les Presses de l'Université de Montréal

ISSN

0026-0452 (imprimé)

Découvrir la revue

Citer cet article

Depecker, L. (1991). Traitement d'une paire terminologique : Ground Range Image et Slant Range Image. Meta, 36(1), 231-233. d'utilisation que vous pouvez consulter en ligne. 


\title{
TRAITEMENT D'UNE PAIRE TERMINOLOGIQUE: GROUND RANGE IMAGE ET SLANT RANGE IMAGE
}

\author{
LOÏC DEPECKER \\ Délégation générale à la langue française, Paris, France
}

\section{EN HOMMAGE À LA COMITAS}

La transposition de termes d'une langue dans une autre, et donc d'un système de références dans un autre, pose en particulier des questions d'ordre:

- conceptuel : il s'agit de définir exactement le concept en cause, ce dernier n'étant ni toujours, ni intégralement transposable ${ }^{1}$;

- linguistique: les éléments entrant dans la composition des signifiants déterminent d'un point de vue morphologique (forme du signifiant) ou morphosyntaxique (ordre des composants) l'angle particulier selon lequel le signe réfère à son objet.

La fixation des équivalents français des termes angloaméricains ground range image et slant range image, effectuée par la commission ministérielle de terminologie de la télédétection aérospatiale (COMITAS), propose sur ce dernier point un exemple original de structuration morphosyntaxique des composants 2 .

La délimitation du concept sous-tendu par le terme ground range image fut effectuée assez rapidement par la COMITAS qui l'établit de la façon suivante: «Image de radar latéral ambiguë dans laquelle les distances-temps verticales sont enregistrées comme étant des distances-temps horizontales.» Il est utile de rappeler ici que la distance-temps est la distance parcourue par l'onde électromagnétique entre le capteur actif et la cible, distance obtenue par la mesure du temps de parcours de cette onde. Ainsi, l'effet désigné par le terme ground range image tend à faire apparaître, sur une image, des objets télédétectés comme plats, alors qu'ils ont en réalité un relief. Ce phénomène est connu depuis longtemps, notamment dans le domaine des images analogiques comme le sont celles de la photographie classique: des arbres par exemple, photographiés d'un avion, apparaissent, sur les clichés d'une prise de vue à axe vertical, comme couchés.

L'équivalent proposé en première analyse fut image dystopographique, le préfixe dys- étant destiné à rendre l'idée de non-adéquation à. De fait, l'inadéquation relevée semble porter sur la topographie du relief qui se présente de façon déformée sur l'image, ce qui est vertical au sol y apparaissant comme horizontal.

Cependant, le rapprochement effectué avec le cliché photographique soulève une objection: dans le cas d'un cliché photographique, l'analyste est conscient du phénomène de projection au sol présenté par ces objets verticaux, alors que l'image-radar (image numérique) dite dystopographique, faisant croire à ce qui n'est pas, introduit un élément de confusion, devenant par là-même source d'erreurs. D'où, en deuxième analyse, l'idée de remplacer le signifiant image dystopographique par dysimage topographique; le report de l'élément dys- sur le terme image permettait effectivement de faire mieux admettre que c'est bien la nature de l'image qui déforme subrepticement la perspective, non la topographie qui déforme l'image. Cela, d'autant plus que lorsque la déformation porte non plus sur le relief, mais sur la distance par rapport à la cible, le concept qui se présente 
est celui de slant range image: «Image de radar latéral dans laquelle les distances-temps, mesurées en oblique, sont restituées comme des distances-temps horizontales.» Dans ce cas, le phénomène qui intervient porte sur la mauvaise estimation que l'on peut faire de la distance entre le capteur et la cible. L'équivalent initialement retenu par la COMITAS pour ce concept fut celui de dysimage-temps, dysimage topographique venant s'inscrire à ses côtés dans un parallélisme morphologique rigoureux.

Pourtant, il convenait d'analyser plus en détail le phénomène susceptible d'être désigné par le terme dysimage-temps. En réalité, il faut bien voir que dans ce cas aussi l'image reste semblable à elle-même, n'étant ni altérée ni inadéquate (dys-): c'est uniquement le système de mesures et la perspective particulière (oblique) selon laquelle s'effectue la prise de données qui sont sources de confusion. D'où la nécessité de faire porter le sème d'inadéquation à non sur le composant image mais sur celui de temps, sous la forme provisoire d'image dystemporelle. Par la même occasion se trouvait confirmé pour le terme ground range image l'équivalent initialement choisi d'image dystopographique. Aux fins de souligner le fait que c'est bien la reproduction du relief qui est dans ce cas en cause, la définition d'image dystopographique précédemment retenue fut complétée de la manière suivante: «Image de radar latéral dans laquelle les distances-temps verticales sont enregistrées comme étant des distances-temps horizontales, introduisant de ce fait une ambiguité sur la topographie.»

La décision de retenir définitivement l'équivalent image dystopographique n'allait évidemment pas sans relancer le débat sur une forme comme image dystemporelle pour slant range image, malgré le parallélisme morphologique a priori rigoureux entre les deux formes françaises. Étant bien entendu que, dans le cas de slant range image, l'image n'est pas véritablement en cause, il s'agit en effet de savoir si pour ce phénomène c'est le temps qui est en cause, et si le préfixe $d y s$ - est le mieux à même de rendre le sens d'inadéquation à. En fait, le trait pertinent dans le phénomène désigné par slant range image est non pas le temps mais la distance: l'image fausse la distance, distorsion due elle-même à une mauvaise restitution du temps de réponse de l'onde sur la cible. Afin de faire apparaître cette caractéristique, il fut décidé de l'inscrire dans la composition du terme: dans un premier temps fut tenté image dysdistancée qui aurait été le terme le plus cohérent du point de vue morphologique et sémantique, s'agissant d'une image sur laquelle les distances sont mal (dys-) restituées. Sur le plan phonique cependant la séquence dysdistancée n'était pas heureuse, ce qui fit penser également à image dédistancée, mais le préfixe dé- n'indiquait pas assez précisément le fait que la restitution des distances est véritablement perturbée sur l'image. Aussi fut-ce finalement en faveur d'image médistancée qu'un accord définitif se fit, le préfixe mé-indiquant de manière explicite la mauvaise (mé-) appréciation des distances restituées par l'image.

Les deux termes furent officialisés de la manière suivante par l'arrêté du 26 juillet 1988 relatif à l'enrichissement du vocabulaire de la télédétection aérospatiale (Journal officiel du 9 septembre 1988):
image dystopographique, n.f.
Domaine :
Télédétection électromagnétique/Technologie des hyperfréquences (incl. radar).
Définition : Image de radar latéral dans laquelle les distances-temps verticales sont enregistrées comme étant des distances-temps horizontales, introduisant de ce fait une ambiguité sur la topographie.
Voir aussi: distance-temps au sol, image médistancée, radar à visée latérale.
Angloaméricain : ground range image 
innage médistancée, n.f.

Domaine :

Télédétection électromagnétique/Technologie des hyperfréquences (incl. radar).

Définition: $\quad$ Image de radar latéral dans laquelle les distances-temps, mesurées en oblique, sont restituées comme des distances-temps horizontales.

Voir aussi: $\quad$ distance-temps au sol, image dystopographique, radar à visée $\begin{array}{ll}\text { Angloamérale. } & \text { laicain: } \\ \text { slant range image. }\end{array}$

En conclusion, on peut noter pour ces deux concepts un parallélisme morphologique dans chacune des deux langues :

-- ground range image (littéralement : «image à distance au sol») slant range image (littéralement : «image à distance oblique»);

- image dystopographique (littéralement : «image à topographie déformée») image médistancée (littéralement : «image à distance erronée»).

D'une langue à l'autre cependant, le parallélisme morphologique ne s'établit pas de la même façon: les termes américains, établis par juxtaposition d'éléments, sont peu explicites pour le non-spécialiste et intraduisibles en l'état. Les termes français, créés de manière concertée, sont en revanche très motivés et permettent de se représenter rapidement et adéquatement les concepts en cause : cette qualité est d'un grand intérêt, notamment pour la traduction vers d'autres langues, et pour l'enseignement des techniques d'analyse d'images.

Il est possible de voir ainsi, dans ce cas de structuration terminologique, un exemple de la manière dont la langue fixe les idées et met en forme le savoir.

\section{NOTES}

1. Voir aussi sur ce sujet «Cinq notions de télédétection aérospatiale: un exemple de structuration d'un champ terminologique», Meta, 34-2, 1989, p. 280 sq.

2. Cette recherche réalisée par la COMITAS occupa les séances de la commission plénière des 21 mai, 25 juin et 16 septembre 1987. 